\title{
Crystallization of ion clouds in octupole traps: Structural transitions, core melting, and scaling laws
}

\author{
F. Calvo* \\ LASIM, Université Claude Bernard Lyon 1 and CNRS, UMR 5579, 43 Boulevard du 11 Novembre 1918, F69622 \\ Villeurbanne Cedex, France \\ C. Champenois \\ PIIM, UMR 6633, Université de Provence and CNRS, Campus Universitaire de Saint-Jérôme C21, \\ F13397 Marseille Cedex 20, France
}

E. Yurtsever

Koç University, Rumelifeneriyolu, Sariyer, Istanbul 34450, Turkey

(Received 20 August 2009; published 1 December 2009)

\begin{abstract}
The stable structures and melting properties of ion clouds in isotropic octupole traps are investigated using a combination of semianalytical and numerical models, with a particular emphasis at finite-size scaling effects. Small-size clouds are found to be hollow and arranged in shells corresponding approximately to the solutions of the Thomson problem. The shell structure is lost in clusters containing more than a few thousands of ions, the inner parts of the cloud becoming soft and amorphous. While melting is triggered in the core shells, the melting temperature follows the rule expected for three-dimensional dense particles, with a depression scaling linearly with the inverse radius.
\end{abstract}

DOI: 10.1103/PhysRevA.80.063401

PACS number(s): 37.10.-x, 36.40.Ei, 52.27.Jt, 52.27.Gr

\section{INTRODUCTION}

The storage of cold ions in electrostatic and magnetic fields (Penning traps) or in radio-frequency electric fields (Paul traps) has become possible with the advent of laser cooling techniques [1-4]. For few-particle systems in quadrupole traps, this has lead to many applications ranging from high-resolution spectroscopy and optical frequency standards $[5,6]$ to quantum information [7-9] and tests on the possible variations of the fundamental constants $[10,11]$. While these effective harmonic traps allow focusing the ions at the center, a greater number of ions with reduced rf-driven motion can be stored in higher-order confinements, with possible uses as microwave clocks for deep space navigation [12] or to control cold chemical reactions [13] of astrophysical relevance.

Large samples of ions also offer a practical realization of the classical many-body Coulomb problem with interesting collective properties such as phase transitions. Crystallization of ion clouds has been observed in quadrupole traps [14-16], in Penning traps [3,17], and theoretically studied by several groups $[18,19]$. There, ion clouds crystallize into well-defined shells at small sizes [18], and then into the bcc Wigner crystal in samples exceeding about $10^{4}$ ions [19]. Melting can be experimentally triggered by varying the trap parameters [20], and proceeds by separate radial and orientational mechanisms in small clusters [21]. Schiffer [22] reported from molecular-dynamics (MD) simulations that the large clouds melt from the surface, resulting in a melting temperature that varies linearly with inverse cluster radius.

In comparison, the properties of cold ion clouds in higherorder traps are much less documented, despite specific inves-

\footnotetext{
*fcalvo@lasim.univ-lyon1.fr
}

tigations for octupole traps [23,24]. Using various semianalytical and numerical methods, we show in this article that the stable structures of ion clouds in octupole traps are generally made of multiple distinct shells only when the number of ions does not exceed a few thousands. Above this approximate size, layering is progressively lost except in the outermost regions of the cloud. We also found that these clusters melt from the core and, quite unexpectedly, exhibit a depression in the melting point that scales linearly with the cloud radius.

In the next section, the model is described and the structural properties of clusters are investigated. In Sec. III, the finite temperature aspects are covered, and some concluding remarks are finally given in Sec. IV.

\section{MODEL AND STATIC PROPERTIES}

The system we are investigating consists of $N$ identical ions with charge $q$ and mass $m$, confined in an isotropic octupole trap. We denote by $\Omega$ the radiofrequency of the electric field and by $\mathcal{E}(\mathbf{r})$ its amplitude at position $\mathbf{r}$. We assume that the adiabatic approximation holds and that the macromotion is driven by the so-called pseudopotential $\Phi_{\mathrm{T}}(\mathbf{r})$ as

$$
\Phi_{\mathrm{T}}(\mathbf{r})=\frac{q^{2}}{4 m \Omega^{2}}[\mathcal{E}(\mathbf{r})]^{2} .
$$

We further assume that the rf-driven motion that superposes with the macromotion can be neglected for the present purposes. For simplicity of the following analysis, the electric field derives from a purely radial three-dimensional octupole potential $\mathcal{E}=-\operatorname{grad} V$ with $V=V_{0} \times r^{4}, r$ being the distance from the trap center and $V_{0}$ a constant. The pseudopotential 
then scales as $r^{6}$ and the potential energy felt by an assembly of ions can thus be written as

$$
\begin{aligned}
\Phi\left(\left\{\mathbf{r}_{i}\right\}\right) & =\Phi_{\mathrm{T}}\left(\left\{\mathbf{r}_{i}\right\}\right)+\Phi_{\mathrm{C}}\left(\left\{\mathbf{r}_{i}\right\}\right), \\
& =A \sum_{i} r_{i}^{6}+B \sum_{i<j} 1 / r_{i j},
\end{aligned}
$$

where we have denoted by $r_{i}$ and $r_{i j}$ the distances of ion $i$ to the center of the trap and to particle $j$, respectively. The constants $A$ and $B$ in the previous equation can be removed by scaling of the quantities $\mathbf{r} \rightarrow \widetilde{\mathbf{r}}=\gamma \mathbf{r}$ and $\Phi \rightarrow \widetilde{\Phi}=\Phi / \gamma B$, with $\gamma=(A / B)^{1 / 7}$. In the following, reduced units are thus chosen for both distances and energies, which amounts to using $A=B=1$ in Eq. (2).

We have first located the structures that globally minimize Eq. (2) at fixed size $N \leq 200$, employing the basin-hopping Monte Carlo method [25] successfully used in previous related work [26]. The ions arrange into a single spherical thin shell for $N<109$, and two shells above this size. This shell structure can be fruitfully used to predict the minima for larger clouds, assuming that the radial density is written as a sum over $M$ concentric shells of zero thickness. Such a shell model was initially developed for the quadrupole trap [27] and improved for intrashell correlations [28]. The energy to be minimized, $\Phi_{\text {shell }}$, is a function of the radii $\left\{R_{i}\right\}$ of the shells, each shell $i$ carrying $N_{i}$ ions (plus one possible ion at the center, $N_{0}$ ),

$$
\Phi_{\text {shell }}\left(\left\{R_{i}, N_{i}\right\}\right)=\sum_{i=1}^{M} \frac{E_{\mathrm{C}}^{\mathrm{intra}}\left(N_{i}\right)}{R_{i}}+\frac{N_{i}}{R_{i}}\left(R_{i}^{7}+\sum_{j<i} N_{j}\right) .
$$

In the above equation, we have denoted by $E_{\mathrm{C}}^{\mathrm{intra}}(N)$ the intrashell Coulomb energy in which the particles lie on the unit sphere. This energy is minimized at the solutions of the Thomson problem [29], which have been tabulated up to rather large sizes [30],

$$
E_{\mathrm{C}}^{\mathrm{intra}}(N)=E_{\mathrm{Th}}(N) .
$$

Alternatively, for large numbers of ions, an asymptotic expression can be substituted for the intrashell Coulomb energy [31] as

$$
E_{\mathrm{C}}^{\mathrm{intra}}(N)=\frac{N(N-\alpha \sqrt{N})}{2},
$$

where the $\alpha \sqrt{N}$ contribution accounts for intrashell correlations [neglecting correlations in a mean-field approach would yield $N(N-1) / 2]$. Following the recent results of Cioslowski and Grzebielucha [31] the parameter $\alpha$ was taken as 1.10610. The above energy $\Phi_{\text {shell }}\left(\left\{R_{i}, N_{i}\right\}\right)$ can be exactly minimized, resulting in the expression for the radius of shell $i$,

$$
R_{i}=\left[\frac{1}{6}\left(\frac{E_{\mathrm{C}}^{\mathrm{intra}}\left(N_{i}\right)}{N_{i}}+\sum_{j<i} N_{j}\right)\right]^{1 / 7} .
$$

The remaining minimization of $\Phi_{\text {shell }}$ with respect to the $\left\{N_{i}\right\}$ must then be carried out numerically under the constraint of a fixed total number of ions.

When the tabulated optimal Thomson energies are used in place for $E_{\mathrm{C}}^{\mathrm{inta}}(N)$, the minimization problem is variational and provides rigorous upper bounds to the exact values. If the asymptotic expression of Eq. (5) is employed instead, energies lower than the numerically exact minima may be reached due to the approximate nature of this asymptotic form. The optimized energies, outer radius, and shell arrangements obtained from Monte Carlo minimization are listed in Table I for selected cluster sizes. In this table, the predictions of the shell models in which the intrashell energies are either taken from the tabulated Thomson minima [30] (discrete model) or from the asymptotic expression (continuous model) are also given.

Energies, shell radius, and ion arrangements agree very well between the three methods; the shell models sometimes producing differences of \pm 1 ion in the shells for the larger clusters containing 180 ions or more. Energies and radii obtained from the tabulated Thomson minima are in very good agreement with the exact results until two shells are formed above 100 ions. The agreement is not as good when the asymptotic form for the Thomson energies is used, the energy being slightly underestimated. This underestimation suggests that the value of the parameter $\alpha$ used in the shell model, which was taken from extrapolations of the Thomson model to the large sizes regime [31], may be slightly excessive at small sizes. The greater validity of the current value of $\alpha$ to large sizes is also indicated by the relative error between the shell energy and the Monte Carlo data, which for the systems considered in Table I decreases from $0.5 \%$ to less than $0.01 \%$ as the number of ions increases from 10 to 200. Despite this systematic error, we generally find that the exact energy obtained by minimization lies in between the predictions of the two shell models, and that the outer radius is correctly reproduced (within $0.2 \%$ ) by both models.

At this point, it is important to stress that the agreement between the shell models and global optimization is essentially due to the correct account of correlations, which are implicitly included in the Thomson energies $E_{\mathrm{Th}}(N)$ or explicitly in the asymptotic expression of Eq. (5) through the term $\delta N=\alpha \sqrt{N}$. For comparison, the mean-field treatment with $\delta N=1$ predicts that the structure of the 100-ion cluster would have nine shells.

The asymptotic shell model can be further exploited in the larger sizes regime $10^{3} \leq N \leq 10^{5}$, where the optimal Thomson energies are not systematically available. The shell radii predicted by this model are represented as a function of the number of ions in Fig. 1. The average radii obtained from globally optimized structures, superimposed for selected sizes $N=10^{k}, k=1-4$, correctly match Eq. (6) until about 1000 ions are reached, larger clouds showing clear deviations in the inner shells. However, the cloud radius (outermost shell) and the minimum energy are both accurately reproduced. One prediction of the continuous shell model is the size at which new shells appear. In contrast with the quadrupole case, where shells are essentially added over an existing core $[18,19]$, shells for octupole clusters grow both on the outside and on the inside. The shell capacitance may then be defined as the maximum number of ions that a shell can sustain, and above which a new shell appears. The continuous shell model predicts the onset of new shells at $N=109, N=442, N=1129, N=2264, N=3992, N=6466$, $N=9709, N=13967$, and $N=19249$. The model also predicts 
TABLE I. Lowest energy $E_{N}$ and outer-shell radius $R_{N}$ found for ion clusters in the octupole trap, as obtained from Monte Carlo global minimization (left columns), from the discrete (Thomson) or continuous (asymptotic) shell models (central and right columns, respectively). The ion arrangements into shells predicted by the three methods are also indicated.

\begin{tabular}{|c|c|c|c|c|c|c|c|c|c|}
\hline \multirow[b]{2}{*}{ Size } & \multicolumn{3}{|c|}{ Minimization } & \multicolumn{3}{|c|}{ Thomson shell model } & \multicolumn{3}{|c|}{ Asymptotic shell model } \\
\hline & Energy & Outer radius & Arrangement & Energy & Outer radius & Arrangement & Energy & Outer radius & Arrangement \\
\hline 10 & 41.624 & 0.917 & $(10)$ & 41.624 & 0.917 & (10) & 41.399 & 0.916 & (10) \\
\hline 20 & 170.363 & 1.033 & $(20)$ & 170.363 & 1.033 & (20) & 170.026 & 1.033 & $(20)$ \\
\hline 30 & 380.045 & 1.104 & $(30)$ & 380.045 & 1.104 & $(30)$ & 379.611 & 1.104 & (30) \\
\hline 40 & 666.975 & 1.156 & $(40)$ & 666.975 & 1.156 & $(40)$ & 666.467 & 1.156 & $(40)$ \\
\hline 50 & 1028.596 & 1.197 & $(50)$ & 1028.596 & 1.197 & $(50)$ & 1027.998 & 1.197 & $(50)$ \\
\hline 60 & 1462.912 & 1.231 & $(60)$ & 1462.912 & 1.231 & $(60)$ & 1462.210 & 1.231 & $(60)$ \\
\hline 70 & 1968.278 & 1.261 & (70) & 1968.278 & 1.261 & (70) & 1967.484 & 1.261 & (70) \\
\hline 80 & 2543.311 & 1.287 & (80) & 2543.311 & 1.287 & (80) & 2542.467 & 1.287 & (80) \\
\hline 90 & 3186.982 & 1.310 & (90) & 3186.983 & 1.310 & (90) & 3185.995 & 1.310 & (90) \\
\hline 100 & 3898.102 & 1.331 & (100) & 3898.103 & 1.331 & (100) & 3897.051 & 1.331 & (100) \\
\hline 110 & 4675.652 & 1.355 & $(2,108)$ & 4675.858 & 1.355 & $(2,108)$ & 4674.619 & 1.355 & $(2,108)$ \\
\hline 120 & 5518.467 & 1.375 & $(4,116)$ & 5518.839 & 1.376 & $(4,116)$ & 5517.415 & 1.374 & $(3,117)$ \\
\hline 130 & 6425.708 & 1.395 & $(6,124)$ & 6426.152 & 1.396 & $(6,124)$ & 6424.708 & 1.396 & $(6,124)$ \\
\hline 140 & 7396.731 & 1.413 & $(8,132)$ & 7397.271 & 1.414 & $(8,132)$ & 7395.796 & 1.414 & $(8,132)$ \\
\hline 150 & 8430.934 & 1.433 & $(12,138)$ & 8431.678 & 1.433 & $(12,138)$ & 8430.054 & 1.432 & $(11,139)$ \\
\hline 160 & 9527.713 & 1.448 & $(14,146)$ & 9528.564 & 1.449 & $(14,146)$ & 9526.851 & 1.447 & $(13,147)$ \\
\hline 170 & 10686.607 & 1.464 & $(17,153)$ & 10687.544 & 1.464 & $(17,153)$ & 10685.664 & 1.463 & $(16,154)$ \\
\hline 180 & 11906.937 & 1.479 & $(20,160)$ & 11907.934 & 1.477 & $(18,162)$ & 11905.975 & 1.478 & $(19,161)$ \\
\hline 190 & 13188.284 & 1.492 & $(22,168)$ & 13189.315 & 1.492 & $(22,168)$ & 13187.303 & 1.492 & $(22,168)$ \\
\hline 200 & 14530.226 & 1.506 & $(26,174)$ & 14531.342 & 1.504 & $(24,176)$ & 14529.198 & 1.506 & $(25,175)$ \\
\hline
\end{tabular}

that the $10^{5}$-ion cluster should have 17 shells but, as will be seen below, structural minimization yields a more contrasted picture. These transitions occur at much larger sizes than in the quadrupole case $[18,19]$, where for instance the 13- and

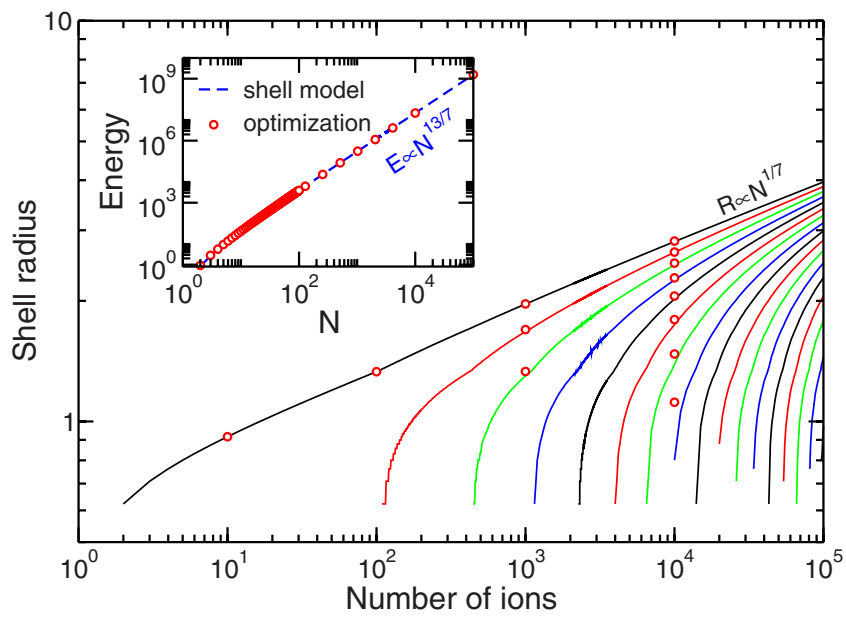

FIG. 1. (Color online) Shell radii versus cluster size, as predicted from the correlated shell model (solid lines; different curves correspond to successive shells) and from global optimization (open circles). The largest shell radius follows a $N^{1 / 7}$ scaling law. Inset: lowest energy versus cluster size, as obtained from the correlated shell model (dashed line) and from global optimization (open circles). 58-ion clusters adopt the $(1,12)$ and $(1,12,45)$ shell structures, respectively [32].

Up to 108 ions, the most stable configurations are the same as the Thomson minima $[29,30]$, within some minor radial relaxations. The Thomson shell model also performs rather well for predicting structures of clusters containing two shells. This agreement suggests using the known Thomson minima for optimizing the atomic structure of multishell systems as well, as long as all shells remain very thin. Such an idea is also supported by a recent investigation by Cioslowski on a modified but similar Thomson model [33]. We have guided Monte Carlo global minimization by combining the results of the shell model with the available solutions to the Thomson problem [30]. In this approach, the continuous shell model is used to predict the optimal number of layers and the individual numbers of ions per layer (the discrete shell model could also be used). The coordinates of the ions in each layer are then scaled from the corresponding solution to the Thomson problem to have a radius given by the predictions of the shell model, and the only remaining degrees of freedom are two Euler angles $\left\{\theta_{i}, \phi_{i}\right\}$ for each layer. The new Monte Carlo optimization consists thus in first locating the low-energy regions in these angles space, and to locally minimize the resulting structures by relaxing all ionic positions. This method was found to perform very satisfactorily with respect to brute force basin-hopping minimization for several sizes in the range $N=100-1000$, leading to low-energy structures often identical, or higher but by only a few $10^{-3}$ percents. 


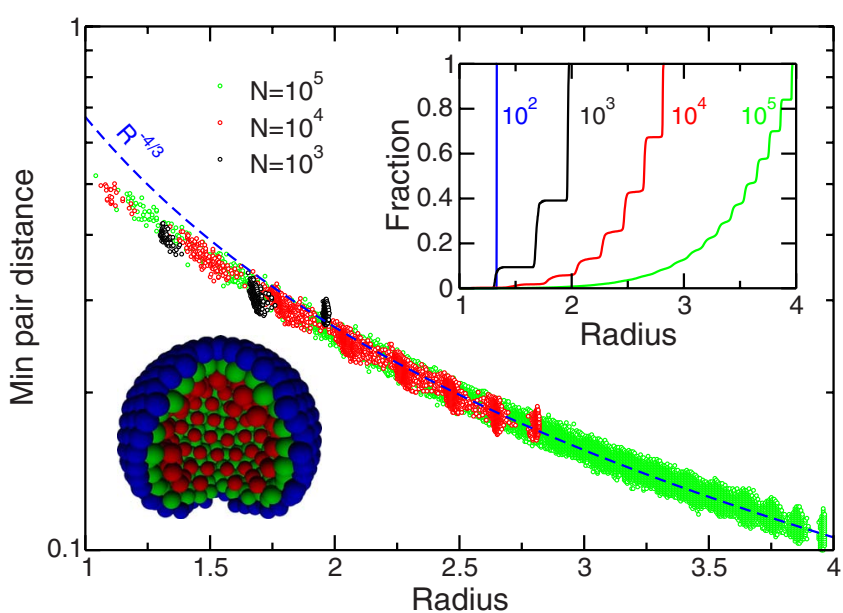

FIG. 2. (Color online) Minimum pair distance versus radial distance in stable structures containing $10^{3}-10^{5}$ ions. Inset: fraction of ions inside a sphere of given radius. The most stable configuration of the 1000-ion cluster is also depicted, with the front quarter removed.

Provided that correlations are included in the evaluation of $E_{\mathrm{C}}^{\text {intra }}(N)$, the above results show that the combined shell model + Monte Carlo optimization should be especially useful for large systems, for which successful basinhopping runs would be prohibitive, and this even allowed us to explore clusters containing up to $N=10^{5}$ ions. At large sizes, Fig. 1 shows that the cloud radius $R$ and its minimum energy $\Phi$ scale as $R \propto N^{1 / 7}$ and $\Phi \propto N^{13 / 7}$, respectively. Both scaling laws readily follow from a simple cold fluid approximation, which is expected to be valid for large sizes [34,35]. The maximum radius $R$ of the cloud naturally relates to the total number $N$ of ions through a $n(r) \propto r^{4}$ radial density. For the energy $\Phi$, the Coulomb and trapping components are related to each other through the virial theorem as $\Phi_{\mathrm{C}}$ $=6 \Phi_{\mathrm{T}}$

at any local minimum configuration where the gradient vanishes. Hence $\Phi$ is proportional to the trapping energy only, which is simply integrated over the radial density $n(r)$ as $\Phi=7 \Phi_{\mathrm{T}} \propto R^{13} \propto N^{13 / 7}$

The validity of the cold fluid theory is better manifested on the radial effective density profile. From the stable ionic configurations obtained for the sizes $N=10^{k}, k=3-5$, we have calculated the minimum pair distance $r_{i j}^{\min }$ between a given ion $i$ and all other ions $j$, which is related to the local density $n(r)$ through $r_{i j}^{\min } \sim n^{-1 / 3}$. Figure 2 shows the correlation between this quantity and the radial distance $r_{i}$ of ion $i$ for the three sizes. The $r^{-4 / 3}$ dependence highlighted in Fig. 2 thus confirms that the cold fluid theory holds increasingly well for these ion clouds, deviations being more noticeable in the less populated (but more fluctuating) inner regions. The most striking feature of Fig. 2 is the broadening of the shells when the number of ions increases from $10^{3}$ to $10^{4}$, especially noticeable for the inner shells. Above a few thousands of ions (an estimate based on mixed-shell-Thomson optimization yields $N^{*} \sim 4000$ ), this broadening is sufficient for some shells to overlap into a more continuous radial distribution of ions below the outermost layer, which is the only one to remain thin, even for the largest size considered,

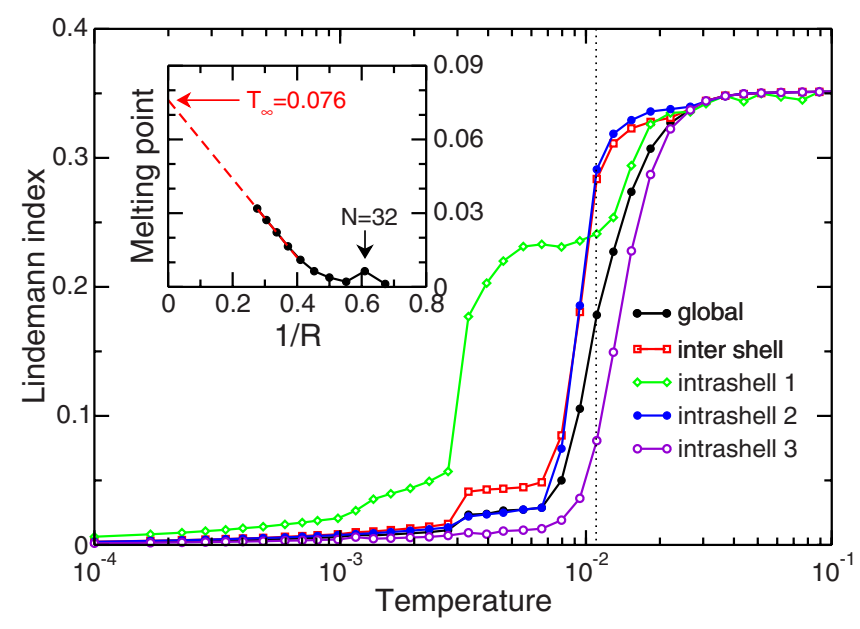

FIG. 3. (Color online) Relative bond-length fluctuation indices obtained for the 512-ion cluster from molecular dynamics simulations. The dotted vertical line marks the onset of the melting temperature for this cluster $\left(T_{m} \simeq 1.1 \times 10^{-2}\right)$. Inset: dependence of the melting temperature with inverse cluster radius, and extrapolation to the infinitely large limit based on results obtained for $N \geq 512$.

$N=10^{5}$. This mixed continuous or discrete behavior is better seen on the accumulated fraction $\chi(r)$ of ions inside a sphere of radius $r$. The variations in $\chi$ with increasing $r$, depicted as an inset in Fig. 2, exhibit spectacular changes as the size reaches $10^{4}$. The sharp steps found in small clouds or at large radii are characteristic of new shells, but are progressively softened in the inner regions of the $10^{4}$-ion system and even replaced by a nearly continuous profile in the largest cluster. In an octupole trap, crystallization should then be understood as the formation of an outermost thin layer with a softer decreasingly dense but thick inner layer. Surface effects, which play a major role in reducing the melting point of ion clouds in harmonic potentials [22], could thus have a very different influence in the case of the octupole trap.

\section{FINITE TEMPERATURE PROPERTIES}

Whereas the previous section considered the stable structures and static issues, we now discuss the thermodynamical and dynamical behavior of selected ion clusters in octupole traps. Classical MD simulations have been carried out at various energies to compute several thermodynamical and structural observables. Order-disorder transitions have been monitored using the root-mean-square bond-length fluctuation or Lindemann index $\delta$, as well as the particle-resolved diffusion constant $D_{i}$ from the integrated velocity autocorrelation function. The rms bond-length fluctuation index has been computed for collective parts of the clouds (intrashell indices) or from pairs of ions belonging to different shells in the stable structure (intershell index). The variations in the shell-resolved Lindemann indices with the kinetic temperature are shown in Fig. 3 for the 512-ion system made of three shells. This size is small enough for the inner shells to be well defined and not overlapping with each other. All Lindemann indices exhibit smooth variations at low and high temperatures, and one sharp increase above $\delta>0.15$ in a narrow 


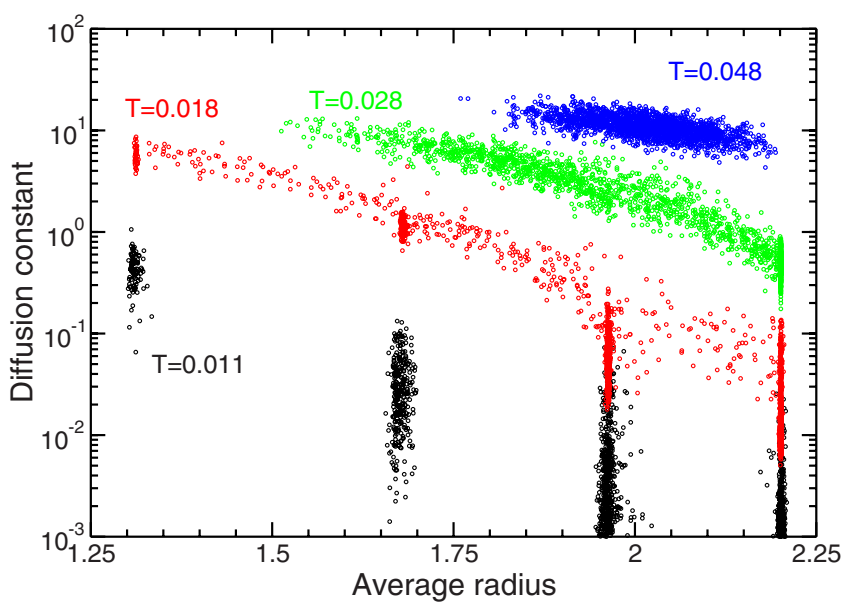

FIG. 4. (Color online) Ion diffusion constant as a function of its time average radius, in the 2048-ion cluster at four temperatures.

temperature range, allowing an accurate estimation of the corresponding melting temperature. From Fig. 3, the global melting of the 512-ion system can therefore be located near $T_{m} \simeq 1.1 \times 10^{-2}$. However, the additional Lindemann indices clearly reveal that the interior shell exhibits some preliminary softening already above $T \simeq 10^{-3}$ before fully melting near $T_{m}^{(1)} \simeq 3 \times 10^{-3}$. Melting of this inner shell impacts the distance fluctuations within the other shells, as seen from the slight increase in the corresponding Lindemann indices. However, these shells clearly undergo their own distinct melting transitions at $T_{m}^{(2)} \simeq 8.5 \times 10^{-3}$ (second shell) and $T_{m}^{(3)} \simeq 1.4 \times 10^{-2}$ (outermost shell). The intershell Lindemann index follows similar variations as the intrashell index of the intermediate second shell. This agreement is not fortuitous: melting of the second shell occurs while the interior shell is already disordered, and the present results show that these two shells tend to mix, to some extent, while the outermost layer remains rigid.

The melting mechanisms can be analyzed in more details by looking at the ions motion at various temperatures. The correlations between the average radial distance $\left\langle r_{i}\right\rangle$ and the diffusion constant $D_{i}$ are shown in Fig. 4 for the 2048-ion system at four characteristic temperatures. For this system, the melting point obtained from the variations in the global Lindemann index lies near $T_{m} \simeq 0.023$. At $T=0.011$, the four shells are clearly seen as narrow vertical spots with low values for $D_{i}$ at radii close to $1.32,1.68,1.97$, and 2.20 . The intershell motion and occasional hops of ions between neighboring shells are seen at $T=0.018$; they are associated with a much higher diffusion constant. Note that $D_{i}$ exhibits a steady decrease with the radial distance, in agreement with the previously found softening of internal layers. At $T=0.028$ the global diffusivity is also higher, and the shell structure seems essentially lost. A similar trend is found at the highest temperature considered here, $T=0.048$, with a single broad spot centered around a radius of 2.02. The peculiar core melting effect is a consequence of the lower density of the inner shells, and is similar in this respect to the general surface melting process in solid state and nanoscale materials [36]. At first sight, this phenomenon may preclude from unambiguously defining the melting temperature of the entire system since, strictly speaking, the system is not yet fully melted when the global Lindemann index barely exceeds 0.15 . However, because the relative number of ions in the external regions grows with size as $N^{1 / 7}$ according to the cold fluid model, the definition of the global index $\delta$ should reflect more and more closely the value of the outermost layer. The melting point $T_{m}$ was thus defined for all sizes as the temperature at which $\delta$ exceeds 0.15 . For the present clouds in octupole traps, the variations in $T_{m}$ have been represented in the inset of Fig. 3 as a function of inverse cluster radius. Not surprisingly, fluctuations are seen at small sizes, and the 32 -ion system $(1 / R \simeq 0.61)$ seems extra resistant to melting relative to its neighboring sizes. Since the 31 - and 33-ion systems have a much lower melting point, the special stability of the 32-ion cluster indicates a magic character, further confirmed by its high icosahedral symmetry. As $N$ reaches $256(1 / R \simeq 0.45), T_{m}$ increases linearly as the inverse radius decreases. This linear depression, though not anticipated for these highly heterogeneous core-melted systems, allows some straightforward extrapolation to the infinite size limit $1 / R \rightarrow 0$, leading to $T_{\infty} \simeq 0.076$. Even though it is hard to figure out what a cloud confined in an octupole trap would physically represent at the bulk limit, the present simulation results should be very useful for estimating melting temperatures in system sizes of the order of $10^{5}$ or more, as experimentally studied in other groups [15].

\section{CONCLUDING REMARKS}

Despite their obvious differences, ion clouds in octupole and quadrupole confinements share several remarkable features. First, the onset of the transition from a multishell structure to the bcc Wigner crystal in quadrupole trap was estimated to be around $10^{4}$ ions [19]. In octupole traps the shell structure becomes blurred above a few thousands of ions. Second, for both cases, the depression in the melting point with respect to the bulk limit follows a linear scaling with inverse cluster radius [22]. Third, in small systems, a clear differential melting between the inner and outer shells seems to take place in both confinements. These similarities may well extend past the specific octupole trap, hold also in higher-order traps, and even be universal. As the exponent $p=6$ in Eq. (2) takes higher integer values, the stable shell structure should be qualitatively preserved, only with fewer shells. Therefore the transition to a softened core should be delayed as $p$ increases, but one cannot exclude that the same melting mechanisms will remain, and in particular that the melting temperature will display linear variations with inverse radius.

Because ion clouds in octupole traps remain poorly studied, the present work could be extended along many lines. Keeping the isotropic case as a model of a more realistic three-dimensional trap, it would be interesting to examine more specifically dynamical properties involving the vibrations and normal modes $[37,38]$ or the transitions between regular and chaotic regimes $[39,40]$. Extension of analytical models $[31,33]$ developed for the quadrupole case to higherorder traps would also be useful.

Finally, at the price of introducing additional parameters in the model, one natural step beyond the present work 
would be to look at clusters in linear octupole traps, such as those discussed by Okada and co-workers [24]. In these systems, confinement along the symmetry axis is harmonic in nature, whereas confinement perpendicular to the axis is octupolar. The stable structures are hollow tubes of ions [24], and it is unclear how the combined contributions of the harmonic and octupolar confinements will determine the cluster properties. Even aware of these complications, the multishell structure and core-melted phase predicted here, as well as the scaling laws connecting size, radius, and melting temperature, should all become amenable to experimental comparison in the near future. May such measurements also help us understanding the collective properties of these exotic states of Coulombic matter.
Note added in proof. Recently, Okada and coworkers, Ref. [41], have observed crystallized ion clouds in linear octupole traps.

\section{ACKNOWLEDGMENTS}

This work was supported under Grant No. ANR-JCJC0053-01. E.Y. would like to acknowledge the support of the Turkish Academy of Sciences. Molecular-dynamics simulations have been carried out at the Pôle Scientifique de Modélisation Numérique, which is gratefully acknowledged.
[1] W. Neuhauser, M. Hohenstatt, P. E. Toschek, and H. G. Dehmelt, Phys. Rev. Lett. 41, 233 (1978).

[2] D. J. Wineland, R. E. Drullinger, and F. L. Walls, Phys. Rev. Lett. 40, 1639 (1978).

[3] W. M. Itano, J. J. Bollinger, J. N. Tan, B. Jelenković, X.-P. Huang, and D. J. Wineland, Science 279, 686 (1998).

[4] T. B. Mitchell, J. J. Bollinger, D. H. E. Dubin, X.-P. Huang, W. M. Itano, and R. H. Baughman, Science 282, 1290 (1998).

[5] T. Rosenband et al., Science 319, 1808 (2008).

[6] M. Chwalla et al., Phys. Rev. Lett. 102, 023002 (2009).

[7] D. Kielpinski, B. E. King, C. J. Myatt, C. A. Sackett, Q. A. Turchette, W. M. Itano, C. Monroe, D. J. Wineland, and W. H. Zurek, Phys. Rev. A 61, 032310 (2000).

[8] F. Schmidt-Kaler, H. Häffner, M. Riebe, S. Gulde, G. P. T. Lancaster, T. Deutschle, C. Becher, C. Roos, J. Eschner, and R. Blatt, Nature (London) 422, 408 (2003).

[9] D. Leibfried et al., Nature (London) 422, 412 (2003).

[10] S. Bize et al., Phys. Rev. Lett. 90, 150802 (2003).

[11] T. M. Fortier et al., Phys. Rev. Lett. 98, 070801 (2007).

[12] J. Prestage, S. Chung, T. Le, L. Lim, and L. Maleki, in Proceedings of IEEE International Frequency Control Symposium, Miami, June 5-7, 2006 (IEEE, New York, 2006).

[13] R. Otto, J. Mikosch, S. Trippel, M. Weidemüller, and R. Wester, Phys. Rev. Lett. 101, 063201 (2008).

[14] F. Diedrich, E. Peik, J. M. Chen, W. Quint, and H. Walther, Phys. Rev. Lett. 59, 2931 (1987).

[15] M. Drewsen, C. Brodersen, L. Hornekær, J. S. Hangst, and J. P. Schiffer, Phys. Rev. Lett. 81, 2878 (1998).

[16] A. Mortensen, E. Nielsen, T. Matthey, and M. Drewsen, Phys. Rev. Lett. 96, 103001 (2006).

[17] S. L. Gilbert, J. J. Bollinger, and D. J. Wineland, Phys. Rev. Lett. 60, 2022 (1988).

[18] R. Rafac, J. P. Schiffer, J. S. Handst, D. H. Dubin, and D. J. Wales, Proc. Natl. Acad. Sci. U.S.A. 88, 483 (1991).

[19] H. Totsuji, T. Kishimoto, C. Totsuji, and K. Tsuruta, Phys. Rev.
Lett. 88, 125002 (2002).

[20] L. Hornekaer, N. Kjaergaard, A. M. Thommesen, and M. Drewsen, Phys. Rev. Lett. 86, 1994 (2001).

[21] J. A. Drocco, C. J. Olson Reichhardt, C. Reichhardt, and B. Jankó, Phys. Rev. E 68, 060401(R) (2003).

[22] J. P. Schiffer, Phys. Rev. Lett. 88, 205003 (2002).

[23] J. Walz, I. Siemers, M. Schubert, W. Neuhauser, R. Blatt, and E. Teloy, Phys. Rev. A 50, 4122 (1994).

[24] K. Okada, K. Yasuda, T. Takayanagi, M. Wada, H. A. Schuessler, and S. Ohtani, Phys. Rev. A 75, 033409 (2007).

[25] D. J. Wales and J. P. K. Doye, J. Phys. Chem. A 101, 5111 (1997).

[26] F. Calvo and E. Yurtsever, Eur. Phys. J. D 44, 81 (2007).

[27] R. W. Hasse and V. V. Avilov, Phys. Rev. A 44, 4506 (1991).

[28] K. Tsuruta and S. Ichimaru, Phys. Rev. A 48, 1339 (1993).

[29] J. J. Thomson, Philos. Mag. 7, 237 (1904).

[30] D. J. Wales and S. Ulker, Phys. Rev. B 74, 212101 (2006).

[31] J. Cioslowski and E. Grzebielucha, Phys. Rev. E 78, 026416 (2008).

[32] R. A. Beekman, M. R. Roussel, and P. J. Wilson, Phys. Rev. A 59, 503 (1999).

[33] J. Cioslowski, Phys. Rev. E 79, 046405 (2009).

[34] D. H. E. Dubin and T. M. O’Neil, Rev. Mod. Phys. 71, 87 (1999).

[35] C. Champenois, J. Phys. B 42, 154002 (2009).

[36] U. Tartaglino, T. Zykova-Timan, F. Ercolessi, and E. Tosatti, Phys. Rep. 411, 291 (2005).

[37] D. H. E. Dubin, Phys. Rev. Lett. 66, 2076 (1991).

[38] G. Téllez, Phys. Rev. E 55, 3400 (1997).

[39] P. Gaspard, Phys. Rev. E 68, 056209 (2003).

[40] E. Yurtsever, F. Calvo, and D. J. Wales, Phys. Rev. E 72, 026110 (2005).

[41] K. Okada, T. Takayanagi, M. Wada, S. Ohtani, and H. A. Schuessler, Phys. Rev. A 80, 043405 (2009). 